\title{
Auxetics among 6-constant tetragonal crystals
}

\author{
R.V. Goldstein, V.A. Gorodtsov, D.S. Lisovenko ${ }^{\dagger}$, M.A. Volkov \\ †lisovenk@ipmnet.ru
}

Institute for Problems in Mechanics RAS, prospect Vernadskogo 101-1, 119526 Moscow, Russia.

Analytical and numerical features of the elastic properties of the stretched rectilinearly anisotropic 6-constant tetragonal crystals are considered. Analytical formulas for Young's modulus and Poisson's ratio are obtained. They are expressed in terms of the elastic compliance coefficients in Voight notation and the parameters of crystal orientation. Numerical calculations are performed using these formulas and data on the elastic constants from the Landolt-Börnstein Handbook. Possible types of Young's modulus and Poisson's ratio are analyzed. More than eighty tetragonal crystals were studied. About $60 \%$ of them are characterized by negative Poisson's ratio for certain particular orientations of the crystals. Such auxetics are listed in the Tables. Ten crystals can have Poisson's ratio greater than unity, and Poisson's ratio for six crystals is less than -0.5 . The same six crystals are characterized by high variability of Young's modulus. Young's modulus for more than ten crystals exceeds $300 \mathrm{GPa}$.

Keywords: Poisson's ratio, Young's modulus, auxetics, tetragonal crystals.

\section{Introduction}

Rectilinear anisotropic crystals have anomalous thermomechanical properties. In particular, for certain orientations, they can have negative Poisson's ratio being auxetics. Auxetics among 6-constant tetragonal crystals, considered below, are studied in the case of rectilinear anisotropy. Previously, similar studies have been performed for all (auxetics and non-auxetics) crystals of cubic and 7-constant tetragonal systems [1-8].

\section{Stretching of crystals with a rectilinear 6-constant tetragonal anisotropy}

Features of such elastic characteristics as Young's modulus and Poisson's ratio in uniaxial tension of crystals depend upon the type of symmetry and orientation of the stretching axis relative to the axes of the crystallographic coordinate system. This orientation is conveniently described by the orthonormal vectors $\mathbf{n}, \mathbf{m},[\mathbf{n m}]$ (n - unit vector along the stretching axis, $\mathbf{m}$ - unit vector orthogonal to the axis of tension) or the three Euler angles $\phi, \theta, \psi$. If in the laboratory coordinate system the axis of elongation corresponds to $(0,0,1)^{\mathrm{T}}$, and a transverse axes correspond to $(1,0,0)^{\mathrm{T}}$ and $(0,1,0)^{\mathrm{T}}$, they will be expressed in terms of Euler's angles in the initial crystallographic coordinate system, as follows:

$$
\begin{gathered}
\mathbf{n}=\left(\begin{array}{c}
\sin \phi \sin \theta \\
-\cos \phi \sin \theta \\
\cos \theta
\end{array}\right), \quad \mathbf{m}=\left(\begin{array}{c}
\cos \phi \cos \psi-\sin \phi \cos \theta \sin \psi \\
\sin \phi \cos \psi+\cos \phi \cos \theta \sin \psi \\
\sin \theta \sin \psi
\end{array}\right), \\
{[\mathbf{n m}]=\left(\begin{array}{c}
-\sin \phi \cos \theta \cos \psi-\cos \phi \sin \psi \\
\cos \phi \cos \theta \cos \psi-\sin \phi \sin \psi \\
\sin \theta \cos \psi
\end{array}\right) .}
\end{gathered}
$$

Young's modulus and Poisson's ratio of 6-constant tetragonal crystals are expressed in terms of the components of the orientation vectors $\mathbf{n}, \mathbf{m}$ and Voigt's matrix compliance coefficients $s_{11}=s_{22}, s_{12}, s_{13}=s_{23}, s_{33}, s_{44}=s_{55}, s_{66}$ as follows:

$$
\begin{gathered}
\frac{1}{\mathrm{E}}=\mathrm{s}_{11}-2 \Delta_{1} \mathrm{n}_{1}^{2} \mathrm{n}_{2}^{2}-2 \Delta_{2} \mathrm{n}_{3}^{2}+\Delta_{3} \mathrm{n}_{3}^{4} \\
\Delta_{1} \equiv \mathrm{s}_{11}-\mathrm{s}_{12}-0.5 \mathrm{~s}_{66}, \quad \Delta_{2} \equiv \mathrm{s}_{11}-\mathrm{s}_{13}-0.5 \mathrm{~s}_{44} \\
\Delta_{3} \equiv \mathrm{s}_{11}+\mathrm{s}_{33}-2 \mathrm{~s}_{13}-\mathrm{s}_{44}, \\
-\frac{v}{\mathrm{E}}=\mathrm{s}_{11}\left(\mathrm{n}_{1}^{2} \mathrm{~m}_{1}^{2}+\mathrm{n}_{2}^{2} \mathrm{~m}_{2}^{2}\right)+\mathrm{s}_{12}\left(\mathrm{n}_{1}^{2} \mathrm{~m}_{2}^{2}+\mathrm{n}_{2}^{2} \mathrm{~m}_{1}^{2}\right)+ \\
+\mathrm{s}_{13}\left(\mathrm{n}_{3}^{2}+\mathrm{m}_{3}^{2}-2 \mathrm{n}_{3}^{2} \mathrm{~m}_{3}^{2}\right)++\mathrm{s}_{33} \mathrm{n}_{3}^{2} \mathrm{~m}_{3}^{2}+ \\
+\mathrm{s}_{44}\left(\mathrm{n}_{1} \mathrm{~m}_{1}+\mathrm{n}_{2} \mathrm{~m}_{2}\right) \mathrm{n}_{3} \mathrm{~m}_{3}+\mathrm{s}_{66} \mathrm{n}_{1} \mathrm{n}_{2} \mathrm{~m}_{1} \mathrm{~m}_{2}
\end{gathered}
$$

In the case of the angular parameterization we have, respectively

$$
\begin{gathered}
\frac{1}{\mathrm{E}}=\mathrm{s}_{11}-\frac{\Delta_{1}}{2} \sin ^{2} 2 \phi \sin ^{4} \theta-2 \Delta_{2} \cos ^{2} \theta+\Delta_{3} \cos ^{4} \theta \\
-\frac{v}{\mathrm{E}}=\mathrm{A}(\phi, \theta) \cos ^{2} \psi+\mathrm{B}(\phi, \theta) \sin ^{2} \psi+\mathrm{D}(\phi, \theta) \sin \psi \cos \psi \\
\mathrm{A}(\phi, \theta)=\mathrm{s}_{12} \sin ^{2} \theta+\mathrm{s}_{13} \cos ^{2} \theta+\frac{\Delta_{1}}{2} \sin ^{2} 2 \phi \sin ^{2} \theta \\
\mathrm{B}(\phi, \theta)=\mathrm{s}_{13}+\frac{1}{4}\left(\Delta_{3}-\frac{\Delta_{1}}{2} \sin ^{2} 2 \phi\right) \sin ^{2} 2 \theta \\
\mathrm{D}(\phi, \theta)=0.5 \Delta_{1} \sin 4 \phi \sin ^{2} \theta \cos \theta
\end{gathered}
$$

Here, Young's modulus is a periodic function with periods $\mathrm{T}_{\phi}=\pi / 2, \mathrm{~T}_{\theta}=\pi$, and Poisson's ratio has periods $\mathrm{T}_{\phi}=\pi / 2$, $\mathrm{T}_{\theta}=2 \pi, \mathrm{T}_{\psi}=\pi$.

Number of studied 6-constant tetragonal crystals is quite large. Information about the elastic properties of 85 such crystals is collected in [9]. Numerical analysis of Poisson's ratio using the above formulas and data on the elastic 
coefficients from [9] showed that about sixty percent of the partial auxetics (crystals having negative Poisson's ratio only for certain directions of orientation) belong to the 6-constant tetragonal crystals.

Maximum, minimum and average of angular values of Poisson's ratios were calculated for all these tetragonal crystals (auxetics and nonauxetics). The extreme characteristics of Poisson's ratios for these crystals were evaluated by a level set method. The results are collected in Table 1. In the same Table, in addition to such global extreme values the extreme values for the three particular orientations of crystals in which the tensile direction vector $\mathbf{n}$ has only one non-zero component are shown for comparison. In rare cases, local extrema coincide with the global extrema. They are highlighted in the Tables in bold. In the cases when the coefficients of elastic compliance were measured at a constant electric field, at a constant electric displacement or a constant polarization we use the notations $\mathrm{s}^{\mathrm{E}}, \mathrm{s}^{\mathrm{D}}, \mathrm{s}^{\mathrm{P}}$, respectively.

In similar Table 2, we performed a comparison of the same global extrema with extreme values of Poisson's ratio for other specific directions with pairs of non-zero components. In these cases, there are much more matches of global and local extrema. Matching values are also labeled in bold.

It can be seen from Table 1, that Poisson's ratio of the several auxetic crystals of 6-constant tetragonal system is less than -0.5. It holds for the crystals: $\mathrm{FeGe}_{2}\left(\nu_{\min }=-0.77\right)$, $\mathrm{Hg}_{2} \mathrm{Br}_{2}\left(v_{\text {min }}=-1.02\right), \mathrm{Hg}_{2} \mathrm{Cl}_{2}\left(v_{\text {min }}=-0.91\right), \mathrm{Hg}_{2} \mathrm{I}_{2}\left(v_{\text {min }}=\right.$ $-0.96),\left(\mathrm{NH}_{2}\right)_{2} \mathrm{CO}\left(v_{\min }=-0.8 ;-0.98\right), \mathrm{TeO}_{2}\left(v_{\min }=-0.8 ;-\right.$ $0.85)$.

The maximum values of Poisson's ratio can exceed unity for such auxetic crystals as In, In-Cd, In-5 at\% Pb, In-Tl, $\mathrm{Hg}_{2} \mathrm{Br}_{2}, \mathrm{Hg}_{2} \mathrm{Cl}_{2}, \mathrm{Hg}_{2} \mathrm{I}_{2}, \mathrm{TeO}_{2},\left(\mathrm{NH}_{2}\right)_{2} \mathrm{CO}$. According to Table 1 only one tetragonal crystal has negative average Poisson's ratio $(\langle v\rangle=-0.13)$.

The results of the calculation of the limits of Young's modulus variation with the change in the stretching direction for all 6-constant tetragonal auxetic crystals show, that the highest variability in Young's modulus (with $\mathrm{E}_{\max } / \mathrm{E}_{\min }>10$ ) have crystals with Poisson's ratio less then -0.5: $\mathrm{Hg}_{2} \mathrm{Br}_{2}\left(\mathrm{E}_{\max } / \mathrm{E}_{\min } \approx 30\right)$, $\mathrm{Hg}_{2} \mathrm{Cl}_{2}\left(\mathrm{E}_{\text {max }} / \mathrm{E}_{\text {min }}=24\right), \quad \mathrm{Hg}_{2} \mathrm{I}_{2}\left(\mathrm{E}_{\text {max }} / \mathrm{E}_{\text {min }} \approx 35\right), \quad \mathrm{TeO}_{2}$ $\left(\mathrm{E}_{\max } / \mathrm{E}_{\min } \approx 13-14\right),\left(\mathrm{NH}_{2}\right)_{2} \mathrm{CO}\left(\mathrm{E}_{\max } / \mathrm{E}_{\min } \geq 12\right), \mathrm{FeGe}_{2}$ $\left(\mathrm{E}_{\max } / \mathrm{E}_{\min } \geq 11\right)$. Young's modulus exceeds $300 \mathrm{GPa}$ for the following crystals: $\mathrm{LuPO}_{4}\left(\mathrm{E}_{\max }=307.7 \mathrm{GPa}\right)$, $\mathrm{BaTiO}_{3}$ with $\mathrm{s}^{\mathrm{D}}\left(\mathrm{E}_{\max } \approx 250-325 \mathrm{GPa}\right), \mathrm{CoPt}\left(\mathrm{E}_{\max }=337.2 \mathrm{GPa}\right)$, $\mathrm{SnO}_{2}\left(\mathrm{E}_{\max }=368.3 \mathrm{GPa}\right), \mathrm{TiO}_{2}\left(\mathrm{E}_{\max }=384.6 \mathrm{GPa}\right), \mathrm{ZrSiO}_{4}$ $\left(\mathrm{E}_{\text {max }}=400 \mathrm{GPa}\right), \mathrm{GeO}_{2}\left(\mathrm{E}_{\max }=465.1 \mathrm{GPa}\right), \mathrm{MoSi}_{2}\left(\mathrm{E}_{\max }=\right.$ 487.6 GPa $), \mathrm{WSi}_{2}\left(\mathrm{E}_{\max }=529.1 \mathrm{GPa}\right)$, Stishovite $\left(\mathrm{E}_{\max }=654.3\right.$ $\mathrm{GPa}), \mathrm{PdPb}_{2}\left(\mathrm{E}_{\max }=684.2 \mathrm{GPa}\right)$.

For partial auxetics the angular regions with negative Poisson's ratio are separated from the regions with a positive coefficient by "auxetic surfaces" with equation of the form $v$ $(\phi, \theta, \psi)=0$. Three types of crystals can be distinguished on the basis of the topological structure of such surfaces. Crystals with "closed" auxetic surfaces form the most extensive group which include $\mathrm{LuPO}_{4}, \mathrm{LuAsO}_{4}, \mathrm{KH}_{2} \mathrm{AsO}_{4}, \mathrm{CdGeAs}_{2}, \mathrm{KH}_{2} \mathrm{PO}_{4}$, $\mathrm{GeO}_{2}$, In-Cd, In-5 at $\% \mathrm{~Pb}$, In-Tl, $\mathrm{Sn}, \mathrm{SnO}_{2}, \mathrm{TiO}_{2}, \mathrm{FeF}_{2}$, $\mathrm{MnF}_{2}, \mathrm{MgF}_{2}, \mathrm{ZnF}_{2}, \mathrm{CoF}_{2}, \mathrm{AgGaS}_{2}, \mathrm{NH}_{4} \mathrm{H}_{2} \mathrm{AsO}_{4}, \mathrm{NH}_{4} \mathrm{H}_{2} \mathrm{PO}_{4}$, $\mathrm{ND}_{4} \mathrm{D}_{2} \mathrm{PO}_{4}, \mathrm{CsH}_{2} \mathrm{AsO}_{4}, \mathrm{RbH}_{2} \mathrm{AsO}_{4}, \mathrm{RbD}_{2} \mathrm{AsO}_{4}, \mathrm{RbH}_{2} \mathrm{PO}_{4}$, $\mathrm{Hg}_{2} \mathrm{Br}_{2}, \mathrm{Hg}_{2} \mathrm{Cl}_{2}$. "Open" surface have, for example, crystals
$\mathrm{BaClF}, \mathrm{FeGe}_{2}, \mathrm{Hg}_{2} \mathrm{I}_{2}, \mathrm{TeO}_{2},\left(\mathrm{NH}_{2}\right)_{2} \mathrm{CO}$. The third least group is formed of the crystals with the auxetic surface of the "mixed" type, which include $\mathrm{InBi}$ and $\mathrm{Li}_{2} \mathrm{~B}_{4} \mathrm{O}_{7}$. Examples of three different types of surfaces for 6-constant tetragonal crystals $\mathrm{SnO}_{2}, \mathrm{BaClF}, \mathrm{Li}_{2} \mathrm{~B}_{4} \mathrm{O}_{7}$ are shown in Figure 1.
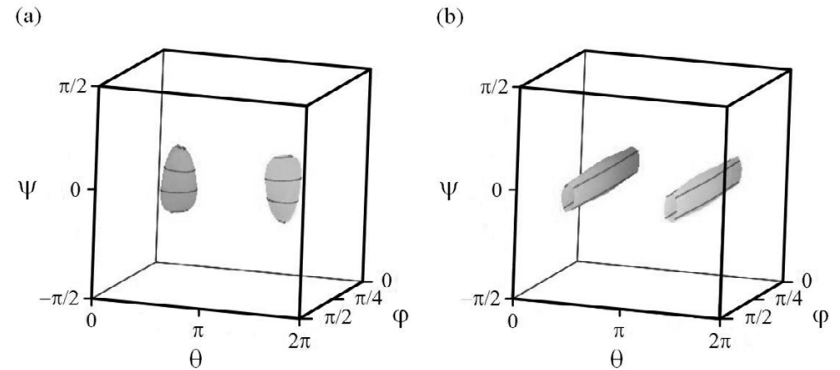

(c)

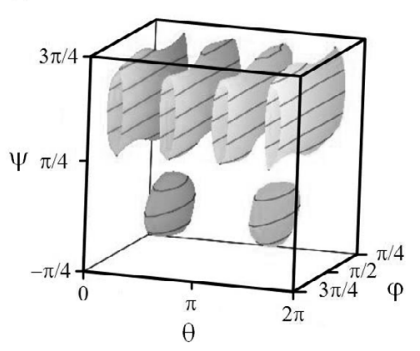

Fig. 1. View of auxetic surfaces for the 6-constant tetragonal crystals $\mathrm{SnO}_{2}(\mathrm{a}), \mathrm{BaClF}(\mathrm{b}), \mathrm{Li}_{2} \mathrm{~B}_{4} \mathrm{O}_{7}$ (c).

\section{Conclusions}

Crystals of 6-constant tetragonal system were considered. In cases when stretching direction coincides with one of the crystallographic axes extrema of Poisson's ratio were found analytically. Values of global minimum and maximum of Poisson's ratio and its angular average value were computed by the numerical analysis on the basis of experimental data. Materials with negative Poisson's ratio and materials for which global extrema coincide with extrema at particular orientations were found. One crystal $\left(\mathrm{FeGe}_{2}\right)$ with negative average Poisson's ratio was revealed. Derived lists of 6-constant tetragonal auxetics considerably extend number of auxetic materials which in the future can find a various applications.

Acknowledgements. The work was supported by the Program for the Basic Research 4.11 of the Department of Energetic, Machinery, Mechanics and Control Processes of the Russian Academy of Sciences, and grant by the President of the Russian Federation for state support of young Russian scientists MK-5891.2015.1, by the Program for Supporting Leading Scientific Schools of Russia NSh-1275.2014.1.

\section{References}

1. R.V. Goldstein, V.A. Gorodtsov, D.S. Lisovenko. Physica status solidi (b), 250 (10), 2038 (2013). DOI: 10.1002/pssb.201384233. 
2. R.V. Goldstein, V.A. Gorodtsov, D.S. Lisovenko, M. A. Volkov. Physical Mesomechanics, 17 (2), 97 (2014). DOI: $10.1134 /$ S1029959914020027.

3. R. V. Goldstein, V. A. Gorodtsov, D. S. Lisovenko. Physical Mesomechanics, 18, (2015).

4. R.H. Baughman, J.M. Shacklette, A.A. Zakhidov, S. Stafström. Nature, 392, 362 (1998). DOI: 10.1038/32842.

5. A.C. Branka, D.M. Heyes, K.W. Wojciechowski. Physica status solidi (b), 246 (9), 2063 (2009). DOI: 10.1002/pssb.200982037.

6. K. W. Wojciechowski. Comput. methods sci. technology, 11 (1), 73 (2005). DOI: 10.12921/cmst.2005.11.01.73 - 79

7. A.N. Norris. Proceedings of the Royal Society of London A. 462, 3385 (2006).

8. V.N. Belomestnykh, E. G. Soboleva. Letters on Materials, 1 (2), 84 (2011). (in Russian).

9. Landolt-Börnstein. Group III: Crystal and Solid State Physics. Vol.29a. (Berlin. Springer, 1992).

Table 1. The extremal and average values of Poisson's ratios of 6-constant tetragonal auxetic crystals. Comparison with local extrema for the cases of one-component vectors orientations of the tensile crystals

\begin{tabular}{|c|c|c|c|c|c|c|c|c|c|}
\hline \multirow{2}{*}{\multicolumn{2}{|c|}{ Crystal }} & \multirow{2}{*}{$v_{\min }$} & \multirow{2}{*}{$v_{\max }$} & \multirow{2}{*}{$<v>$} & \multicolumn{2}{|c|}{ at $\mathrm{n}_{1}=1$} & \multicolumn{2}{|c|}{ at $\mathrm{n}_{2}=1$} & \multirow{2}{*}{$\begin{array}{l}v \text { at } \\
\mathrm{n}_{3}=1\end{array}$} \\
\hline & & & & & $v_{\min }$ & $v_{\max }$ & $v_{\min }$ & $v_{\max }$ & \\
\hline \multirow{2}{*}{\multicolumn{2}{|c|}{$\mathrm{NH}_{4} \mathrm{H}_{2} \mathrm{AsO}_{4}$}} & -0.06 & 0.66 & 0.35 & -0.06 & 0.66 & -0.06 & 0.66 & 0.26 \\
\hline & & -0.34 & 0.73 & 0.33 & -0.34 & 0.73 & -0.34 & 0.73 & 0.29 \\
\hline \multicolumn{2}{|l|}{$\mathrm{NH}_{4} \mathrm{H}_{2} \mathrm{AsO}_{4} 44 \%$ deuterated } & -0.46 & 1.10 & 0.35 & -0.46 & 1.10 & -0.46 & 1.10 & 0.36 \\
\hline \multicolumn{2}{|l|}{$\mathrm{NH}_{4} \mathrm{H}_{2} \mathrm{PO}_{4}$} & -0.12 & 0.66 & 0.34 & -0.12 & 0.66 & -0.12 & 0.66 & 0.27 \\
\hline $\mathrm{ND}_{4} \mathrm{D}_{2} \mathrm{PO}_{4}$ & $S^{E}$ & -0.11 & 0.59 & 0.32 & -0.11 & 0.58 & -0.11 & 0.58 & 0.25 \\
\hline \multicolumn{2}{|l|}{$\mathrm{BaClF}$} & -0.05 & 0.73 & 0.31 & -0.04 & 0.72 & -0.04 & 0.72 & 0.35 \\
\hline \multirow{2}{*}{$\mathrm{BaTiO}_{3}$} & $\mathrm{~S}^{\mathrm{E}}$ & -0.13 & 1.04 & 0.38 & 0.29 & 0.65 & 0.29 & 0.65 & 0.33 \\
\hline & $\mathrm{s}^{\mathrm{E}}$ & -0.18 & 0.98 & 0.35 & 0.19 & 0.67 & 0.19 & 0.67 & 0.33 \\
\hline \multirow{2}{*}{\multicolumn{2}{|c|}{$\mathrm{CdGeAs}_{2}$}} & -0.09 & 0.78 & 0.33 & 0.33 & 0.48 & 0.33 & 0.48 & 0.39 \\
\hline & & -0.05 & 0.73 & 0.33 & 0.35 & 0.45 & 0.35 & 0.45 & 0.38 \\
\hline \multicolumn{2}{|l|}{$\mathrm{CsH}_{2} \mathrm{AsO}_{4}$} & -0.11 & 0.88 & 0.32 & 0.01 & 0.03 & 0.01 & 0.03 & 0.03 \\
\hline \multicolumn{2}{|l|}{$\mathrm{CoF}_{2}$} & -0.12 & 0.69 & 0.34 & 0.15 & 0.64 & 0.15 & 0.64 & 0.38 \\
\hline \multicolumn{2}{|l|}{$\mathrm{GeO}_{2}$} & -0.12 & 0.51 & 0.26 & 0.17 & 0.47 & 0.17 & 0.47 & 0.36 \\
\hline \multirow{2}{*}{\multicolumn{2}{|c|}{ In }} & -0.42 & 1.31 & 0.45 & 0.31 & 0.64 & 0.31 & 0.64 & 0.48 \\
\hline & & -0.71 & 1.64 & 0.47 & -0.07 & 1.04 & -0.07 & 1.04 & 0.49 \\
\hline \multicolumn{2}{|l|}{ InBi } & -0.21 & 0.97 & 0.37 & 0.35 & 0.61 & 0.35 & 0.61 & 0.36 \\
\hline \multicolumn{2}{|l|}{ In-3.4 at $\% \mathrm{Cd}$} & -0.32 & 1.22 & 0.45 & 0.46 & 0.50 & 0.46 & 0.50 & 0.47 \\
\hline \multicolumn{2}{|l|}{ In-3.42 at\% Cd } & -0.33 & 1.22 & 0.45 & 0.47 & 0.49 & 0.47 & 0.49 & 0.47 \\
\hline \multicolumn{2}{|l|}{ In-5 at $\% \mathrm{~Pb}$} & -0.30 & 1.17 & 0.45 & 0.21 & 0.75 & 0.21 & 0.75 & 0.44 \\
\hline \multicolumn{2}{|l|}{ In-10 at $\% \mathrm{Tl}$} & -0.47 & 1.35 & 0.46 & 0.43 & 0.53 & 0.43 & 0.53 & 0.49 \\
\hline \multicolumn{2}{|l|}{ In-11.5 at $\% \mathrm{Tl}$} & -0.41 & 1.32 & 0.46 & 0.40 & 0.56 & 0.40 & 0.56 & 0.48 \\
\hline \multicolumn{2}{|l|}{ In-15 at $\% \mathrm{Tl}$} & -0.48 & 1.38 & 0.46 & 0.46 & 0.50 & 0.46 & 0.50 & 0.48 \\
\hline \multicolumn{2}{|l|}{$\mathrm{FeF}_{2}$} & -0.13 & 0.70 & 0.36 & 0.19 & 0.62 & 0.19 & 0.62 & 0.42 \\
\hline $\mathrm{FeGe}_{2}$ & & -0.77 & 0.39 & -0.13 & -0.04 & 0.39 & -0.04 & 0.39 & 0.03 \\
\hline $\mathrm{Pb}_{0,346} \mathrm{Ba}_{0,59} \mathrm{Na}_{0.036} \mathrm{Li}_{0,028}-\mathrm{Nb}_{2} \mathrm{O}_{6}$ & E & -0.04 & 0.35 & 0.07 & -0.03 & 0.35 & -0.03 & -0.04 & 0.35 \\
\hline $\mathrm{Li}_{2} \mathrm{~B}_{4} \mathrm{O}_{7}$ & $S^{E}$ & -0.15 & 0.61 & 0.15 & -0.13 & 0.61 & -0.13 & -0.15 & 0.61 \\
\hline $\mathrm{LuAsO}_{4}$ & & -0.08 & 0.80 & 0.32 & -0.02 & 0.32 & -0.02 & 0.32 & 0.34 \\
\hline $\mathrm{LuPO}_{4}$ & & -0.11 & 0.83 & 0.33 & 0.01 & 0.30 & 0.01 & 0.30 & 0.32 \\
\hline $\mathrm{MgF}_{2}$ & & -0.01 & 0.57 & 0.28 & 0.13 & 0.57 & 0.13 & 0.57 & 0.27 \\
\hline $\mathrm{MnF}_{2}$ & & -0.07 & 0.75 & 0.36 & 0.13 & 0.70 & 0.13 & 0.70 & 0.40 \\
\hline $\mathrm{Hg}_{2} \mathrm{Br}_{2}$ & & -1.02 & 1.94 & 0.40 & 0.02 & 0.90 & 0.02 & 0.90 & 0.61 \\
\hline $\mathrm{Hg}_{2} \mathrm{Cl}_{2}$ & & -0.91 & 1.75 & 0.40 & 0.02 & 0.91 & 0.02 & 0.91 & 0.43 \\
\hline $\mathrm{HgI}_{2}$ & & -0.11 & 0.80 & 0.34 & -0.11 & 0.80 & -0.11 & 0.80 & 0.31 \\
\hline $\mathrm{Hg}_{2} \mathrm{I}_{2}$ & & -0.96 & 1.98 & 0.40 & 0.03 & 0.88 & 0.03 & 0.88 & 0.88 \\
\hline $\mathrm{NiF}_{2}$ & & -0.04 & 0.71 & 0.35 & 0.13 & 0.68 & 0.13 & 0.68 & 0.36 \\
\hline $\mathrm{KD}_{2} \mathrm{AsO}_{4}$ & & -0.07 & 0.64 & 0.37 & -0.07 & 0.52 & -0.07 & 0.52 & 0.39 \\
\hline
\end{tabular}


Table 1. (cont.)

\begin{tabular}{|c|c|c|c|c|c|c|c|c|c|}
\hline \multirow{2}{*}{\multicolumn{2}{|c|}{ Crystal }} & \multirow{2}{*}{$v_{\min }$} & \multirow{2}{*}{$v_{\max }$} & \multirow{2}{*}{$<v>$} & \multicolumn{2}{|c|}{ at $\mathrm{n}_{1}=1$} & \multicolumn{2}{|c|}{ at $\mathrm{n}_{2}=1$} & \multirow{2}{*}{$\begin{array}{c}v \text { at } \\
\mathrm{n}_{3}=1\end{array}$} \\
\hline & & & & & $v_{\min }$ & $v_{\max }$ & $v_{\min }$ & $v_{\max }$ & \\
\hline \multicolumn{2}{|l|}{$\mathrm{KD}_{2} \mathrm{PO}_{4}$} & -0.13 & 0.65 & 0.27 & -0.13 & 0.25 & -0.13 & 0.25 & 0.20 \\
\hline \multicolumn{2}{|l|}{$\mathrm{KH}_{2} \mathrm{AsO}_{4}$} & -0.05 & 0.63 & 0.29 & -0.05 & 0.30 & -0.05 & 0.30 & 0.21 \\
\hline \multicolumn{2}{|l|}{$\mathrm{KH}_{2} \mathrm{PO}_{4}$} & -0.13 & 0.65 & 0.28 & -0.13 & 0.28 & -0.13 & 0.28 & 0.21 \\
\hline$\left(\mathrm{K}_{1 / 6} \mathrm{Na}_{1 / 6} \mathrm{Sr}_{1 / 2} \mathrm{Ba}_{1 / 6}\right) \mathrm{Nb}_{2} \mathrm{O}_{6}$ & $\mathrm{~s}^{\mathrm{D}}$ & -0.16 & 0.33 & 0.05 & -0.15 & 0.33 & -0.15 & 0.33 & -0.12 \\
\hline \multicolumn{2}{|l|}{$\mathrm{RbD}_{2} \mathrm{AsO}_{4}$} & -0.41 & 0.56 & 0.19 & -0.41 & 0.18 & -0.41 & 0.18 & 0.16 \\
\hline \multicolumn{2}{|l|}{$\mathrm{RbH}_{2} \mathrm{AsO}_{4}$} & -0.37 & 0.58 & 0.16 & -0.37 & 0.08 & -0.37 & 0.08 & 0.07 \\
\hline \multicolumn{2}{|l|}{$\mathrm{RbH}_{2} \mathrm{PO}_{4}$} & -0.14 & 0.76 & 0.29 & -0.14 & 0.23 & -0.14 & 0.23 & 0.18 \\
\hline \multicolumn{2}{|l|}{$\mathrm{AgGaS}_{2}$} & -0.06 & 0.83 & 0.38 & 0.29 & 0.55 & 0.29 & 0.55 & 0.40 \\
\hline \multicolumn{2}{|l|}{ Stishovite } & -0.04 & 0.44 & 0.22 & 0.16 & 0.40 & 0.16 & 0.40 & 0.31 \\
\hline \multirow{2}{*}{\multicolumn{2}{|c|}{$\mathrm{TeO}_{2}$}} & -0.80 & 1.49 & 0.35 & 0.02 & 0.91 & 0.02 & 0.91 & 0.23 \\
\hline & & -0.85 & 1.55 & 0.35 & 0.02 & 0.92 & 0.02 & 0.92 & 0.22 \\
\hline \multirow{2}{*}{\multicolumn{2}{|c|}{ TlSe }} & -0.22 & 0.66 & 0.28 & -0.22 & 0.53 & -0.22 & 0.53 & 0.66 \\
\hline & & -0.18 & 0.49 & 0.18 & -0.10 & 0.45 & -0.10 & 0.45 & 0.35 \\
\hline \multicolumn{2}{|l|}{$\mathrm{Sn}$} & -0.03 & 0.80 & 0.36 & 0.10 & 0.76 & 0.10 & 0.76 & 0.29 \\
\hline \multicolumn{2}{|l|}{$\mathrm{SnO}_{2}$} & -0.11 & 0.62 & 0.30 & 0.14 & 0.59 & 0.14 & 0.59 & 0.35 \\
\hline \multicolumn{2}{|l|}{$\mathrm{TiO}_{2}$} & -0.03 & 0.68 & 0.29 & 0.13 & 0.59 & 0.13 & 0.59 & 0.33 \\
\hline \multirow{2}{*}{\multicolumn{2}{|c|}{$\left(\mathrm{NH}_{2}\right)_{2} \mathrm{CO}$}} & -0.80 & 1.89 & 0.44 & -0.17 & 0.53 & -0.17 & 0.53 & 0.78 \\
\hline & & -0.98 & 1.73 & 0.37 & -0.07 & 0.16 & -0.07 & 0.16 & 0.33 \\
\hline \multirow{2}{*}{\multicolumn{2}{|c|}{$\mathrm{ZnF}_{2}$}} & -0.05 & 0.65 & 0.35 & 0.16 & 0.63 & 0.16 & 0.63 & 0.39 \\
\hline & & -0.05 & 0.66 & 0.35 & 0.16 & 0.63 & 0.16 & 0.63 & 0.39 \\
\hline \multicolumn{2}{|l|}{$\mathrm{Zn}\left[\mathrm{C}\left(\mathrm{NH}_{2}\right)_{3}\right]_{2}\left(\mathrm{SO}_{4}\right)_{2}$} & -0.14 & 0.67 & 0.31 & -0.14 & 0.67 & -0.14 & 0.67 & 0.42 \\
\hline
\end{tabular}

Table 2. The extremal and average values of Poisson's ratios and the local extrema in the case of two-component vectors orientations of the tensile 6-constant tetragonal auxetic crystals

\begin{tabular}{|c|c|c|c|c|c|c|c|c|c|c|}
\hline \multirow{2}{*}{\multicolumn{2}{|c|}{ Crystal }} & \multirow{2}{*}{$v_{\min }$} & \multirow{2}{*}{$v_{\max }$} & \multirow{2}{*}{$<v>$} & \multicolumn{2}{|c|}{ at $\mathrm{n}_{1}=0$} & \multicolumn{2}{|c|}{ at $\mathrm{n}_{2}=0$} & \multicolumn{2}{|c|}{ at $\mathrm{n}_{3}=0$} \\
\hline & & & & & $v_{\min }$ & $v_{\max }$ & $v_{\min }$ & $v_{\max }$ & $v_{\min }$ & $v_{\max }$ \\
\hline \multirow{2}{*}{\multicolumn{2}{|c|}{$\mathrm{NH}_{4} \mathrm{H}_{2} \mathrm{AsO}_{4}$}} & -0.06 & 0.66 & 0.35 & -0.06 & 0.66 & -0.06 & 0.66 & -0.06 & 0.66 \\
\hline & & -0.34 & 0.73 & 0.33 & -0.34 & 0.73 & -0.34 & 0.73 & -0.34 & 0.73 \\
\hline \multicolumn{2}{|c|}{$\mathrm{NH}_{4} \mathrm{H}_{2} \mathrm{AsO}_{4} 44 \%$ deuterated } & -0.46 & 1.10 & 0.35 & -0.46 & 1.10 & -0.46 & 1.10 & -0.46 & 1.10 \\
\hline \multicolumn{2}{|c|}{$\mathrm{NH}_{4} \mathrm{H}_{2} \mathrm{PO}_{4}$} & -0.12 & 0.66 & 0.34 & -0.12 & 0.66 & -0.12 & 0.66 & -0.12 & 0.66 \\
\hline $\mathrm{ND}_{4} \mathrm{D}_{2} \mathrm{PO}_{4}$ & $S_{S}^{\mathrm{E}}$ & -0.11 & 0.59 & 0.32 & -0.11 & 0.59 & -0.11 & 0.58 & -0.11 & 0.58 \\
\hline \multicolumn{2}{|c|}{$\mathrm{BaClF}$} & -0.05 & 0.73 & 0.31 & -0.05 & 0.73 & -0.04 & 0.72 & -0.04 & 0.72 \\
\hline \multirow{2}{*}{$\mathrm{BaTiO}_{3}$} & $S^{E}$ & -0.13 & 1.04 & 0.38 & -0.13 & 1.04 & 0.16 & 0.65 & 0.16 & 0.65 \\
\hline & $s^{E}$ & -0.18 & 0.98 & 0.35 & -0.18 & 0.98 & 0.18 & 0.67 & 0.18 & 0.67 \\
\hline \multirow{2}{*}{\multicolumn{2}{|c|}{$\mathrm{CdGeAs}_{2}$}} & -0.09 & 0.78 & 0.33 & -0.09 & 0.78 & -0.08 & 0.68 & -0.08 & 0.68 \\
\hline & & -0.05 & 0.73 & 0.33 & -0.05 & 0.73 & -0.05 & 0.65 & -0.05 & 0.65 \\
\hline \multicolumn{2}{|c|}{$\mathrm{CsH}_{2} \mathrm{AsO}_{4}$} & -0.11 & 0.88 & 0.32 & 0.00 & 0.88 & 0.01 & 0.55 & 0.01 & 0.55 \\
\hline \multicolumn{2}{|c|}{$\mathrm{CoF}_{2}$} & -0.12 & 0.69 & 0.34 & -0.12 & 0.64 & 0.11 & 0.66 & 0.11 & 0.66 \\
\hline \multicolumn{2}{|c|}{$\mathrm{GeO}_{2}$} & -0.12 & 0.51 & 0.26 & -0.12 & 0.47 & 0.09 & 0.51 & 0.09 & 0.51 \\
\hline \multirow{2}{*}{\multicolumn{2}{|c|}{ In }} & -0.42 & 1.31 & 0.45 & -0.42 & 1.31 & -0.01 & 0.91 & -0.01 & 0.91 \\
\hline & & -0.71 & 1.64 & 0.47 & -0.69 & 1.64 & -0.18 & 1.09 & -0.18 & 1.09 \\
\hline \multicolumn{2}{|c|}{ InBi } & -0.21 & 0.97 & 0.37 & -0.05 & 0.97 & -0.21 & 0.80 & -0.21 & 0.80 \\
\hline \multicolumn{2}{|c|}{ In-3.4 at $\%$ Cd } & -0.32 & 1.22 & 0.45 & -0.32 & 1.22 & -0.11 & 1.01 & -0.11 & 1.01 \\
\hline \multicolumn{2}{|c|}{ In-3.42 at $\%$ Cd } & -0.33 & 1.22 & 0.45 & -0.33 & 1.22 & -0.12 & 1.01 & -0.12 & 1.01 \\
\hline \multicolumn{2}{|c|}{ In-5 at $\% \mathrm{~Pb}$} & -0.30 & 1.17 & 0.45 & 0.21 & 0.75 & -0.30 & 1.17 & -0.30 & 1.17 \\
\hline \multicolumn{2}{|c|}{ In-10 at $\% \mathrm{Tl}$} & -0.47 & 1.35 & 0.46 & -0.47 & 1.35 & -0.29 & 1.22 & -0.29 & 1.22 \\
\hline \multicolumn{2}{|c|}{ In-11.5 at $\% \mathrm{Tl}$} & -0.41 & 1.32 & 0.46 & -0.41 & 1.32 & -0.14 & 1.06 & -0.14 & 1.06 \\
\hline \multicolumn{2}{|c|}{ In-15 at $\% \mathrm{Tl}$} & -0.48 & 1.38 & 0.46 & -0.48 & 1.38 & -0.35 & 1.26 & -0.35 & 1.26 \\
\hline
\end{tabular}


Table 2. (cont.)

\begin{tabular}{|c|c|c|c|c|c|c|c|c|c|c|}
\hline \multirow{2}{*}{\multicolumn{2}{|c|}{ Crystal }} & \multirow{2}{*}{$v_{\min }$} & \multirow{2}{*}{$v_{\max }$} & \multirow{2}{*}{$<v>$} & \multicolumn{2}{|c|}{ at $\mathrm{n}_{1}=0$} & \multicolumn{2}{|c|}{ at $\mathrm{n}_{2}=0$} & \multicolumn{2}{|c|}{ at $\mathrm{n}_{3}=0$} \\
\hline & & & & & $v_{\min }$ & $v_{\max }$ & $v_{\min }$ & $v_{\max }$ & $v_{\min }$ & $v_{\max }$ \\
\hline \multicolumn{2}{|l|}{$\mathrm{FeF}_{2}$} & -0.13 & 0.70 & 0.36 & -0.13 & 0.62 & 0.08 & 0.70 & 0.08 & 0.70 \\
\hline \multicolumn{2}{|l|}{$\mathrm{FeGe}_{2}$} & -0.77 & 0.39 & -0.13 & -0.04 & 0.39 & -0.77 & 0.39 & -0.77 & 0.39 \\
\hline $\mathrm{Pb}_{0.346} \mathrm{Ba}_{0.59} \mathrm{Na}_{0.036} \mathrm{Li}_{0.028}-\mathrm{N}$ & $S^{E}$ & -0.04 & 0.35 & 0.07 & -0.04 & 0.35 & -0.03 & 0.35 & -0.03 & 0.35 \\
\hline $\mathrm{Li}_{2} \mathrm{~B}_{4} \mathrm{O}_{7}$ & $S^{E}$ & -0.15 & 0.61 & 0.15 & -0.13 & 0.61 & -0.13 & 0.61 & -0.13 & 0.61 \\
\hline \multicolumn{2}{|l|}{$\mathrm{LuAsO}_{4}$} & -0.08 & 0.80 & 0.32 & -0.02 & 0.72 & -0.02 & 0.44 & -0.02 & 0.44 \\
\hline \multicolumn{2}{|l|}{$\mathrm{LuPO}_{4}$} & -0.11 & 0.83 & 0.33 & 0.01 & 0.74 & 0.01 & 0.44 & 0.01 & 0.44 \\
\hline \multicolumn{2}{|l|}{$\mathrm{MgF}_{2}$} & -0.01 & 0.57 & 0.28 & -0.01 & 0.57 & 0.07 & 0.57 & 0.07 & 0.57 \\
\hline \multicolumn{2}{|l|}{$\mathrm{MnF}_{2}$} & -0.07 & 0.75 & 0.36 & -0.07 & 0.70 & 0.04 & 0.75 & 0.04 & 0.75 \\
\hline \multicolumn{2}{|l|}{$\mathrm{Hg}_{2} \mathrm{Br}_{2}$} & -1.02 & 1.94 & 0.40 & 0.01 & 0.90 & -1.02 & 1.94 & -1.02 & 1.94 \\
\hline \multicolumn{2}{|l|}{$\mathrm{Hg}_{2} \mathrm{Cl}_{2}$} & -0.91 & 1.75 & 0.40 & 0.02 & 0.91 & -0.91 & 1.75 & -0.91 & 1.75 \\
\hline \multicolumn{2}{|l|}{$\mathrm{HgI}_{2}$} & -0.11 & 0.80 & 0.34 & -0.11 & 0.80 & -0.11 & 0.80 & -0.11 & 0.80 \\
\hline \multicolumn{2}{|l|}{$\mathrm{Hg}_{2} \mathrm{I}_{2}$} & -0.96 & 1.98 & 0.40 & -0.19 & 0.88 & -0.96 & 1.99 & -0.96 & 1.99 \\
\hline \multicolumn{2}{|l|}{$\mathrm{NiF}_{2}$} & -0.04 & 0.71 & 0.35 & -0.04 & 0.68 & 0.06 & 0.71 & 0.06 & 0.71 \\
\hline \multicolumn{2}{|l|}{$\mathrm{KD}_{2} \mathrm{AsO}_{4}$} & -0.07 & 0.64 & 0.37 & -0.07 & 0.63 & -0.07 & 0.64 & -0.07 & 0.64 \\
\hline \multicolumn{2}{|l|}{$\mathrm{KD}_{2} \mathrm{PO}_{4}$} & -0.13 & 0.65 & 0.27 & -0.13 & 0.65 & -0.13 & 0.48 & -0.13 & 0.48 \\
\hline \multicolumn{2}{|l|}{$\frac{\mathrm{KH}_{2} \mathrm{AsO}_{4}}{\mathrm{KH} \mathrm{PO}}$} & -0.05 & 0.63 & 0.29 & -0.05 & 0.63 & -0.05 & 0.52 & -0.05 & 0.52 \\
\hline \multicolumn{2}{|l|}{$\mathrm{KH}_{2} \mathrm{PO}_{4}$} & -0.13 & 0.65 & 0.28 & -0.13 & 0.65 & -0.13 & 0.50 & -0.13 & 0.50 \\
\hline$\left(\mathrm{K}_{1 / 6} \mathrm{Na}_{1 / 6} \mathrm{Sr}_{1 / 2} \mathrm{Ba}_{1 / 6}\right) \mathrm{Nb}_{2} \mathrm{O}_{6}$ & $\mathrm{~S}^{\mathrm{D}}$ & -0.16 & 0.33 & 0.05 & -0.16 & 0.33 & -0.15 & 0.33 & -0.15 & 0.33 \\
\hline \multicolumn{2}{|l|}{$\mathrm{RbD}_{2} \mathrm{AsO}_{4}$} & -0.41 & 0.56 & 0.19 & -0.41 & 0.56 & -0.41 & 0.42 & -0.41 & 0.42 \\
\hline \multicolumn{2}{|l|}{$\mathrm{RbH}_{2} \mathrm{AsO}_{4}$} & -0.37 & 0.58 & 0.16 & -0.37 & 0.58 & -0.37 & 0.37 & -0.37 & 0.37 \\
\hline \multicolumn{2}{|l|}{$\mathrm{RbH}_{2} \mathrm{PO}_{4}$} & -0.14 & 0.76 & 0.29 & -0.14 & 0.76 & -0.14 & 0.51 & -0.14 & 0.51 \\
\hline $\mathrm{AgGaS}_{2}$ & & -0.06 & 0.83 & 0.38 & -0.06 & 0.83 & 0.11 & 0.60 & 0.11 & 0.60 \\
\hline Stishovite & & -0.04 & 0.44 & 0.22 & -0.04 & 0.40 & 0.05 & 0.44 & 0.05 & 0.44 \\
\hline $\mathrm{TeO}_{2}$ & & -0.80 & 1.49 & 0.35 & -0.18 & 0.91 & -0.80 & 1.49 & -0.80 & 1.49 \\
\hline & & -0.85 & 1.55 & 0.35 & -0.17 & 0.92 & -0.85 & 1.55 & -0.85 & 1.55 \\
\hline TlSe & & -0.22 & 0.66 & 0.28 & -0.22 & 0.53 & -0.22 & 0.66 & -0.22 & 0.66 \\
\hline & & -0.18 & 0.49 & 0.18 & -0.10 & 0.45 & -0.10 & 0.45 & -0.10 & 0.45 \\
\hline $\mathrm{Sn}$ & & -0.03 & 0.80 & 0.36 & 0.10 & 0.76 & -0.03 & 0.80 & -0.03 & 0.80 \\
\hline $\mathrm{SnO}_{2}$ & & -0.11 & 0.62 & 0.30 & -0.11 & 0.59 & 0.08 & 0.62 & 0.08 & 0.62 \\
\hline $\mathrm{TiO}_{2}$ & & -0.03 & 0.68 & 0.29 & -0.03 & 0.59 & 0.02 & 0.63 & 0.02 & 0.63 \\
\hline$(\mathrm{NH}$ & & 0.10 & 0.25 & 0.15 & 0.11 & 0.25 & 0.10 & 0.25 & 0.10 & 0.25 \\
\hline 2 & & -0.80 & 1.89 & 0.44 & -0.17 & 0.82 & -0.17 & 0.78 & -0.17 & 0.78 \\
\hline $\mathrm{Z}$ & & -0.98 & 1.73 & 0.37 & -0.07 & 0.91 & -0.07 & 0.53 & -0.07 & 0.53 \\
\hline 2 & & 0.20 & 0.26 & 0.23 & 0.20 & 0.26 & 0.20 & 0.26 & 0.22 & 0.26 \\
\hline $\mathrm{Zn}\left[\mathrm{C}\left(\mathrm{NH}_{2}\right)_{3}\right]_{2}\left(\mathrm{SO}_{4}\right)$ & & -0.05 & 0.65 & 0.35 & -0.05 & 0.63 & 0.12 & 0.65 & 0.12 & 0.65 \\
\hline
\end{tabular}

